\title{
Excursionen an die Brüteplätze von Sterna, Larus und Glareola im Golf von Smyrna im Frühling 1859.
}

\author{
Von \\ Guido von Gonzenbach. \\ (Schluss; s. S. $308-316$.)
}

Die Fischer von Chomata hatten sich selbst / verwundert, dass ich noch keine Möven - Eier gefunden und meinten, dass diese Glari sonst in dieser Zeit schon/angefangen Eier zu legen; ich solle in zehn Tagen wiederkommen. Wirklich hatte ich auch den Vorsatz in einigen Tagen wieder nach genannten Fischereien zurückzukehren.

Sowohl die eingetretene veränderliche Witterung, als auch andere Umstände bewirkten, dass ich erst am 21. Juni den beabsichtigten zweiten Besuch zu den Fischereien in Ausführung bringen konnte. Ich bediente/mich der nämlichen Barke, nahm/jedoch nur zwei Ruderer. Das Wetter war schöner und stetiger geworden, als es während einiger Wochen /gewesen und meine Bootsleute meinten, ich dürfte die Fahrt nicht länger verschieben, da die meisten Seeschwalben - und MövenEier sonst vielleicht schon zu sehr bebrütet oder gar ausgekommen seien.

Ich fahre also an genanntem Tage, Abends $10 \mathrm{Uhr}$, bei bewölktem Himmel und schwachem Südwind von Smyrna ab. Der Wind wechselt während der Nacht oft, um Mitternacht scheint der Mond hie und da durchs Gewölke, das nach Norden getrieben wird. Wir halten uns fern von der südlichen Küste, um so wenig Weg als möglich zu machen, müssen aber sehr Acht geben, um nicht zwischen die Sandbänke und Untiefen rechts hineinzugerathen. Endlich wird glücklich um die hervorspringendste Sandbankzunge, die sich von der nördlichen Küste weit in den Golf hineinerstreckt, herumgebogen, und wir fahren bei frischem Sudwind stets mit aufgespanntem Segel, nicht sehr weit von der nördlichen seichten Küste, längs derselben hin, während meine aufmerksamen Griechen im Dunkel und bei neblichter Luft ausspähen, die Salzhügel oder Wohnungen der Salzgewinner zu entdecken suchend, um darnach ihre Fahrt nach den Fischereien zu richten und die Einfahrt nicht zu verfehlen. - Endlich sehen wir im Vorbeifliegen, denn wir fahren bei sehr frischem Winde, an der Kúste in ziemlicher Entfernung ein Paar der grossen Salzladebarken, Cernik genannt, dann kommen wir beim Wrack einer griechischen Brigantine vorbei, wonach wir uns genau orientiren, es war nămlich noch Nacht, ungefahr $2 \frac{1}{2}$ Uhr. Das Segel wird rasch 
eingezogen, aber die Wellen kommen und rollen, vom Südwind anfgeregt, hoch daher, die Brandung tobt auf diesen wenig tiefen Ufern hohl und stark, einen Augenblick ist das Boot nahe daran von einer solchen rollenden Welle in die Quere gefasst und umgeworfen zu werden, glücklicher Weise ändert einer der Leute mit einem kräftigen Rucke des Ruders die Richtung des Bootes, so dass die nachfolgende Welle das Hintertheil trifft und so gegen das Land stösst, in nicht mehr gefährlicher Lage. - Nach einigen Minuten bringen die kräftigen Ruderschläge meiner zwei Leute mich glücklich zu den Strohhütten, wo wir das erstere Mal gewesen. Diese Fischerei wird wie weiter oben gesagt „Chomata “ genannt. Es mag Morgens 3 Uhr sein. Ich steige ans Land und gehe zu einer der Schilfhütten. Dieselbe besteht einfach aus einer im Kreis und im Sande stehenden 8-10 Fuss hohen Schilfwand, die oben nicht zugedeckt ist, aber mit einer Eingangsthür versehen; hieher kommen nun nach und nach ungefähr ein Dutzend Fischer um ihr Nargileh zu rauchen (eine Wasserpfeife, wo der Rauch durch Wasser gehen muss,) und ihren schwarzen Caffé in kleinen Tässchen zu schlürfen, in der Mitte des Kreises lodert ein Feuer. Erst nachdem wir mehr als eine Stunde da gesesselı, wobei im Ganzen kaum einige Worte gesprochen wurden und die Fischer sich wieder entfernt hatten, fragte endlich der Obmann der Fischer, ob wir wegen der Möven-Eier gekommen. Auf unsere bejahende Antwort meinte derselbe, wir sollten diesmal noch weiter in die Fischereien hineinfahren, als die zwei Inseln lägen, auf welchen wir bei meinem ersten Besuche die GlareolaEier gefunden, bis gegen die Salzwerke hinunter, Uspurinia genannt. Dieser Obmann erlaubte uns auch wieder, uns eines der flachen Fischerboote zu bedienen. - Wir nehmen endlich Urlaub, die Sonne war im Osten aufgegangen, meine zwei Leute nehmen jeder eine Stange und einer am Vordertheil der Kurita (Barke) stehend, der andere am Hintertheil, stossen wir endlich vom Ufer. Ich höre und sehe keine Vögel, nur etwa ein Numenius arquatus oder eine Seeschwalbe lassen ihre Stimme hören und letztere fliegt fischend in schnellem Bogenfluge über diese seichten, gelblichen, stillen Gewässer. - Die Sonne war bereits aufgegangen und fing ihre Wärme schon an sich fühlbar zu machen, als wir die Landzunge, wo Salz bereitet wird, und die vom Meer aus sich hier in die Fischereien hineinerstreckt, erreichten. Das Land, eine dunkle lehmige Erde, ist ganz niedrig, kaum zwei Schuh über dem Wasser erhoben und daher durch und durch mit Salzwasser geschwängert, welches hier und da Lachen bildend, und theilweise vertrocknet, eine dicke Salzkruste wie gefrorener Schnee hinterlässt. - Ganz 
niedriges Gesträuch, von Pflanzen, welche nur in solch salzhaltigem Erdreiche fortkommen, gebildet, bedeckt den vorderen Theil dieser Landzunge, wo wir anlanden. Auf diesem feuchten niedrigen Landungsplatze sah ich nur einige Budytes flava (alles B. melanocephala), sie flogen hie und da aus dem Gestrüpp und mögen wohl darin nisten. - In der Entfernung von ein Paar hundert Schritten bemerkte ich einige Männer, beschäftigt das Ufer aufzudämmen, und bald gelangen wir zu einer steinernen länglichen Hütte, welche ganz wohnlich eingerichtet ist; sie gehört den Salzgewinnern, Einer dieser Männer (Grieche) frägt, was wir an diesem abgelegenen Orte suchen, eine begreifliche Frage, da wohl selten ein Europäer diese Oertlichkeit betreten hat, oder vielleicht noch nie, wenigstens zn dem mir vorgenommenen Zwecke. Als ich von Möven-Eiern sprach, welche ich suche, zeigt uns der Mann, zu dem bald ein aufgeweckter Knabe aus der Hütte herbei gelaufen kam, einige zerbrochene Schalen von Seeschwalben-Eiern, die vor der Hütte lagen und wovon der Inhalt von den Salzmännern genossen worden. „Gebt dem , pädi ( Kind) ein kleines Trinkgeld und es wird Euch die Brüteplätze der Glari und Glaraki (Möven und Seeschwalben) zeigen, " sagt der Mann zu uns. Und sogleich, nachdem ich nach Landesart mit dem Kopfe ein zustimmendes Zeichen gemacht, läuft der Knabe behend vor uns her nach einer Gegend weit hinter der Hütte, gegen Westen. Die Erde ist hier, nachdem ein Salzwerk passirt, ganz sandig, trocken und fest, auf Muschelgrund, mit kaum Schuh hohen Pflanzen einer und derselben Art hie und da bedeckt, sonst meist offene Lehde, die sich weit gegen West-Nordwest ausdehnt; gleich hinter dem Salzwerk sehe ich an einem Wasserpfuhl ein Paar Larus argentatus stehen, die aber nicht Stand halten, sondern von Weitem auffliegen. Aber auf der genannten offenen Lehde, wo die Morgensonne fürchterlich brannte, angekommen, sehe ich bald eine Menge Seeschwalben verschiedener Art, besonders St. hirundo und anglica und auch viele Glareola pratincola über//unseren Köpfen herumfliegen. Die letzteren (Gl. pratincola) setzen sich oft auf die Erde, immer, wo ein offener Platz, und nehmen da ăusserst curiose Stellungen an, indem sie die Flügel wie Segel in die Höhe heben; sie halten dabei den Körper aufrecht, nachher dehnen und strecken sie die Flügel ganz aus, in horizontaler Lage, die Spitzen berühren die Erde, legen sich mit Kopf, Hals und Vorderkörper flach auf die Erde und bleiben so mit ausgebreiteten Flügeln ein Paar Secunden liegen. Ich begreife nicht, warum sie, diese Stellung annehmen, wenn nicht, um die Aufmerksamkeit des Menschen auf sich und vom nahen Nistplatz, wo bereits die Jungen sitzen und piepen, abzulenken?! 
Der griechische Knabe, der eine sehr geläufige Zunge hat, ruft mich zu einigen Nestern, wenn man eine kleine Vertiefung, um welche einige trockene Schilfstückchen liegen, so nennen will; denn nur die grösseren Eier, die ich als von St. anglica erkenne, liegen zu dreien in einer Art von Nest, das von ganz verwittertem Schilfrohr oder anderen trockenen Pflanzenhalmen kunstlos, aber kreisrund, wie ein nicht tiefer Trichter gebaut ist. Das Nest liegt nicht etwa verborgen, sondern ganz offen auf kahler Erde, doch kann das ausschlüpfende Junge einen kärglichen Schatten unter den niedrigen Pflänzchen, die nahebei stehen, finden, wie ich selbst gesehen habe; auch bleiben die Jungen nie im Nest oder da, wo die Eier gelegt wurden, sondern sind stets einige Zoll daneben und wo möglich unter den spärlichen Pflanzen versteckt. Ich fand ein Junges von St. anglica, den Schnabel in die Höhe gestreckt und etwas daraus hervorragend, das ich Anfangs für die Zunge hielt, das'sich dann aber als der Schwanz eines Meerpferdehens oder einer ähnlichen Art Meerthiere ergab, als einer meiner Leute daran zog. Ich besah viele Nester und Eier, nahm aber nur wenige Eier mit (nebst einigen Jungen, die ich in Spiritus bewahre,) weil ich vermuthen musste, dass alle stark bebrütet sein müssten, und zudem zu den bereit 5 allbekannten oben genanuten Seeschwalben-Arten gehörten. Leider sah ich keinen einzigen Larus melanocephalus, obwohl durch meine wiederholten Schüsse auf Glareola und Sterna hirundo sich Hunderte von Seeschwalben aufgeschreckt über unseren Häuptern schreiend und kreischend herumtummelten und auf mich stiessen. Nachdem ich mich über eine Stunde an diesem Brüteplatze der Seeschwalben aufgehalten und nebenbei einige im Sand begrabene Muscheln (Dolium galea) gesammelt hatte, gab ich endlich den Bitten der Bootsleute nach, von denen Einer sich schlafen gelegt hatte, trotz der brennenden Sonnenhitze, deren Wirkung ich selbst zu spüren anfing, und machte mich auf den Rückweg. Hier in diesen Hintergewässern geht gar kein Lüftchen, wenn auch draussen auf offenem Meer heftige Winde die Wellen hochgehen machen. Auf der Rückfahrt, die mehr als eine Stunde dauert, betrat ich die Inseln nicht, wo ich auf der ersten Fahrt Glareola-Eier gefunden, da ich gar keinen Vogel darüber hinfliegen sah. Ich kehrte daher direct nach den Schilfhütten zurück, wo ich die Fischer mit dem Binden von Schilfrohrhecken beschäftigt fand. Hier unter diesem offenen Schilfrohrverschlage war es sehr kühl, der Wind bliess heftig von der gegenüberliegenden südlichen Küste, und fühlte ich von den heissen Innengewässern kommend, den Unterschied so stark, dass ich mich in meinen Tuchmantel hüllte. - So war also diese 
zweite Excursion Betreffs Larus melanocephalus ebenfalls fruchtlos ausgefallen, dennoch bereute ich es nicht, noch einmal diese Localitäten besucht zu haben. Wegen der Sturmtaucher-Eier (Puffinus cinereus und anglorum) von den Griechen „Michi ${ }^{\star}$ genannt, konnte mir hier Niemand Auskunft geben, und doch sieht man diese Vögel oft im Golf. Ich nahm nun mein frugales Mahl ein, aus ein wenig kaltem Braten und Brod bestehend und einem Schluck Wein, versuchte vergeblich während der Zeit bis zur Abfahrt nach Smyrna zu schlafen. Um Mittagszeit luden mich die Fischer ein, an ihrer in Wasser gekochten Bohnensuppe :Theil zu nehmen, selbige war jedoch nicht so einladend, wie die das erste Mal servirte Aalsuppe, ich bedankte mich deshalb höflichst. Einer der Fischer brummte dagegen: „Aale giebt es, mein lieber Herr, nicht jedesmal aufzutischen." Endlich rufen mir meine Leute zu, dass es Zeit zum Aufbrechen sei, und nachdem ich mich bei den Fischern die mich so gastfreundlich aufgenommen, verabschiedet, besteige ich wieder mein Caïk, noch einmal einen Blick auf die Gegend, die mir so Interessantes geboten, zurüchwerfend und sie mir tief einprägend; denn kaum werde ich je wieder dahin zurückkehren. Obwohl die Heftigkeit des Windes seit der Nacht eher zugenommen und die Brandung drohend an dieser niederen gefährlichen Küste daher rollte, setzen wir dennoch wohlgemuth das Segel auf und fahren im Flug derselben entlang bis an eine vorspringende Sandbank, einfach ${ }_{\text {Capo }}{ }^{*}$ von den Griechen genannt. Hier muss das Segel gewendet werden, um bei den gegenüber liegenden Inseln wieder günstigen Wind zu holen. Wir kämpfen auf diesem Querweg über den Golf mit sehr hohem Meer, unser kleines Fahrzeug ist oft bedroht von den Wogen überwältigt zu werden, doch kommen wir jedesmal mit einem Staubregen davon. So gegen Wind und Meer ansegelnd, erreichen wir nach fast einer Stunde die Nähe der "Isolá lunga"; von hier wenden wir das Boot von der südlichen in eine östliche Richtung, so dass der Südwind, „Garbi“ von den Griechen geheissen, von der Seite des Segels fassend, und das Boot auf die linke Seite legend, uns gerade nach den „Castelli“ zutreibt, wo er uns auf einmal verlässt und immer schwächer wird. Bis wir nach Smyrna kommen, wird es 5 Uhr Abends. - Ich war nämlich sehr froh auch diese Fahrt glúcklich überstanden zu haben, denn wie die obige Erzählnng dargethan, ist sie nicht ganz ohne Gefahr, der Unannehmlichkeiten einer Nachtfahrt in offenem Boote nicht zu gedenken.

Wie ich schon zu Anfange dieses Berichtes bemerkt habe, sollte man Zeit und Musse haben einige Wochen an diesen Innengewässern und Buchten zu verweilen, und zwar von Mitte Mai bis Ende Juni. 
Meine Stellung und mein Geschäft erlauben mir eine verlängerte $\mathbf{A b}$ wesenheit nicht, daher muss ich es anderen Ornithologen überlassen, diese Gegenden behufs Auffindung der Eier von Larus melanocephalus noch näher zu erforschen; ich habe ihnen einstweilen den Weg gezeigt und einen Leitfaden an die Hand gegeben. - Ein Besuch dieser Küsten, sowohl der südlichen als nördlichen, und selbst bis aus den Golf hinaus im Frühling, dürfte jedenfalls für den Ornithologen, besonders aber für den Oologen von grossem Interesse sein; denn nach den Aussagen meiner Eiersammler nisten an den Küsten, da wo sie steiler abfallen, auch noch andere Vögel als Meerschwalben und Möven : nämlich: Tauben', Falken, Adler, Lämmergeier und andere Arten Vögel. Aber Geduld und Ausdauer und eine gute feste Gesundheit gehören dazu, um die Strapazen während länger als einem Monat auszuhalten, die ein Aufenthalt an diesen öden, unwirthlichen Küsten mit sich bringt.

Ich hoffe hiermit einen kleinen Beitrag zur erweiterten Kenntniss der Brüteplätze einiger Vogelarten geliefert zu haben, welcher von den Lesern dieses Journals mit einigem Interesse aufgenommen werden dürfte. Zugleich bitte ich um Nachsicht wegen der Weitläufigkeit meines Berichts; ich dachte aber es sei besser: derselbe sei etwas lang und deutlich, als kurz und undeutlich.

Smyrna, im August 1859.

KUmatisehe Versehönerung des Gesanges. - In meinem Werkchen über die Einflüsse des Klima's habe ich die Gründe der interessanten Erscheinung angegeben, dass höher im Norden und weiter im 0 sten von uns manche Singvögel entschieden besser singen, als dieselben Arten bei uns, und hier wieder viel besser, als namentlich in England. (Nämlich: die grössere Heiterkeit der Witterung in Sommer des Nordens und die dortige Länge der Tage, so ivie die alsdann fortwährend ungetrübte Heiterkeit des Himmels in den tiefen Binnenländern des Ostens, daher namentlich in Sibirien, stimmen auch die, für solche Eindrücke so vorzugsweise empfängliche Vogelwelt heiterer und fröhlicher, als bei uns, oder gar in dem trüben und nebelreichen Britannien. Die Folge hiervon ist, dass ihr Gesang durch viel häufigere Uebung sich vervollkommnet, dass also stets bessere Lehrmeister darin für die jungen Männchen vorhanden sind, als bei uns, und dass auf diese Weise im Verlaufe unvordenklicher Zeiten die grössere Vollkommenheit eine bleibende, allgemeine geworden ist.) Noch dazu gilt diess yon manchen Vogelarten, deren Gesang wir bei uns nur sehr 


\section{$2 \mathrm{BHL}$ Biodiversity Heritage Library}

Gonzenbach, Guido von. 1859. "Excursionen an die Brüteplätze von Sterna, Larus und Glareola im Golf von Smyrna im Frühling 1859." Journal $f u$

r Ornithologie 7, 393-398. https://doi.org/10.1007/bf02009342.

View This Item Online: $\underline{\text { https://www.biodiversitylibrary.org/item/50195 }}$

DOI: https://doi.org/10.1007/bf02009342

Permalink: https://www.biodiversitylibrary.org/partpdf/141453

\section{Holding Institution}

Natural History Museum Library, London

\section{Sponsored by}

Natural History Museum Library, London

\section{Copyright \& Reuse}

Copyright Status: Public domain. The BHL considers that this work is no longer under copyright protection.

This document was created from content at the Biodiversity Heritage Library, the world's largest open access digital library for biodiversity literature and archives. Visit BHL at https://www.biodiversitylibrary.org. 